\title{
Paramedics experiences and expectations concerning advance directives: A prospective, questionnaire-based, bi-centre study
}

Palliative Medicine 26(7) 908-916

(C) The Author(s) 20II

Reprints and permission:

sagepub.co.uk/journalsPermissions.nav DOI: |0.1|77/02692|63||4|9885

pmj.sagepub.com

\author{
Mahmoud Taghavi University Medical Center Goettingen, Germany \\ Alfred Simon Academy of Medical Ethics, University Medical Center Goettingen, Germany \\ Stefan Kappus Medical director, Emergency Medical Service, Hamburg, Germany \\ Nicole Meyer Department of Anaesthesiology, University Hospital of Regensburg, Germany \\ Christoph L Lassen Department of Anaesthesiology, University Hospital of Regensburg, Germany \\ Tobias Klier Department of Anaesthesiology, University Hospital of Regensburg, Germany \\ David B Ruppert Department of Anaesthesiology and Intensive Care Medicine, Municipal Hospital of Lueneburg, Germany \\ Bernhard M Graf Department of Anaesthesiology, University Hospital of Regensburg, Germany \\ Gerd G Hanekop Department of Anaesthesiology, Emergency and Intensive Care Medicine, University Medical Center Goettingen \\ Christoph HR Wiese Department of Anaesthesiology, University Hospital of Regensburg, Germany
}

\begin{abstract}
Background: Advance directives and palliative crisis cards are means by which palliative care patients can exert their autonomy in end-of-life decisions.

Aim: To examine paramedics' attitudes towards advance directives and end-of-life care.

Design: Questionnaire-based investigation using a self-administered survey instrument.

Setting/participants: Paramedics of two cities (Hamburg and Goettingen, Germany) were included. Participants were questioned as to (I) their attitudes about advance directives, (2) their clinical experiences in connection with end-of-life situations (e.g. resuscitation), (3) their suggestions in regard to advance directives, 'Do not attempt resuscitation' orders and palliative crisis cards.

Results: Questionnaires were returned by 728 paramedics (response rate: 81\%). The majority of paramedics (7I\%) had dealt with advance directives and end-of-life decisions in emergency situations. Most participants (84\%) found that cardiopulmonary resuscitation in end-of-life patients is not useful and $75 \%$ stated that they would withhold cardiopulmonary resuscitation in the case of legal possibility. Participants also mentioned that more extensive discussion of legal aspects concerning advance directives should be included in paramedic training curricula. They suggested that palliative crisis cards should be integrated into end-of-life care.

Conclusions: Decision making in prehospital end-of-life care is a challenge for all paramedics. The present investigation demonstrates that a dialogue bridging emergency medical and palliative care issues is necessary. The paramedics indicated that improved guidelines on end-of-life decisions and the termination of cardiopulmonary resuscitation in palliative care patients may be essential. Participants do not feel adequately trained in end-of-life care and the content of advance directives. Other recent studies have also demonstrated that there is a need for training curricula in end-of-life care for paramedics.
\end{abstract}

\section{Keywords}

Advance directives, palliative care, paramedics, prehospital emergency care

\section{Background}

The right to self-determination is a fundamental human right and is recognized in several national constitutions (e.g. German Constitution: Article 1, Section 1, Part 1), as is the guarantee of human dignity and the right to physical integrity. ${ }^{1}$ For this, 'informed consent' between the patient and health care providers is essential. Discussions and questionnaire-based

Corresponding author:

Christoph H.R. Wiese, MD, PhD, Department of Anaesthesiology, University of Regensburg, Franz-Josef-Strauß-Allee I I, D-93053 Regensburg, Germany. Email: christoph.wiese@klinik.uni-regensburg.de 
investigations in the United States, Canada, and Germany with prehospital emergency physicians and paramedics have underscored the importance of this issue. Internationally, however, it is important that in prehospital emergency medicine, a viable therapeutic approach be implemented based, for example, on the 'four principles of medical ethics' (autonomy, nonmaleficence, beneficence, justice). ${ }^{2}$

An advance directive is defined as an individual written order which declares the patient's will in special circumstances. It is usually implemented through the use of special forms, medical wristbands or necklaces. Accordance to the new German law concerning advance directives, a third person should be appointed to make health care decisions on nonrespondent patients' behalf in accordance with their will. Furthermore, it is worth mentioning that in certain crisis situations, a third person must be appointed. But this fact is not always the case, even though it is recommended by the legislator. Since advance directives are often rather generally formulated, a palliative crisis card (PCC; for example Figure 1, modified from Wiese et al. ${ }^{3}$ ), as is used in health facilities, caregivers and professional health care providers (paramedics) to not attempt resuscitation if the patient is found pulseless. Those emergency sheets (PCCs) are similar to 'DNAR orders' as used in the United States. ${ }^{4}$ With these instruments, the patient's autonomy at the end of life may be strengthened. ${ }^{5}$

In prehospital emergency situations, PCCs were created to prevent futile attempts at cardiopulmonary resuscitation of end-of-life patients. However, the establishment of such sheets requires accurate legal knowledge in all health care providers (paramedics). $6,7,8$

In the present investigation, patients with an incurable disease who were in a far-advanced stage of their disease were defined as 'end-of-life'. Pulselessness is another stage of the end-of-life situation. To make a difference between a crisis situation in a palliative stage of disease and an endof-life one seems to be very difficult. Therefore, it may be helpful to define the patient and his or her individual situation as a 'palliative care situation'.

Palliative emergencies account for about $3-5 \%$ of all prehospital emergency situations. Therefore, every paramedic may have to deal with such an emergency and the special needs of palliative care patients. ${ }^{3}$

Overall, in most countries (e.g. Germany, United Kingdom) it is not legally clear-cut whether paramedics may withhold resuscitation in prehospital emergency medical care (regardless of the existence of an advance directive). Paramedics usually must initiate full resuscitation of patients without vital signs. Due to emergency legal regulations, paramedics must act on the pro-vita principle. This fact is binding even though an advance directive or a PCC exists. Furthermore, an existing advance directive or a 'DNAR order' is often not recognized as a legitimate reason to withhold resuscitation. Paramedics have even less latitude than prehospital emergency physicians. The initiation of resuscitation is exactly what they have to do - regardless of the patient's wishes. Therefore, new guidelines for prehospital end-of-life decision making and the need of education of paramedics and prehospital emergency physicians in this judicial circumstances, seem to be crucial. Also, there is a need for more information and statements by official lawyers. These statements have to be communicated to paramedics and prehospital emergency physicians as well.

A main objective of this investigation was to determine paramedics' practices in regard to withholding and

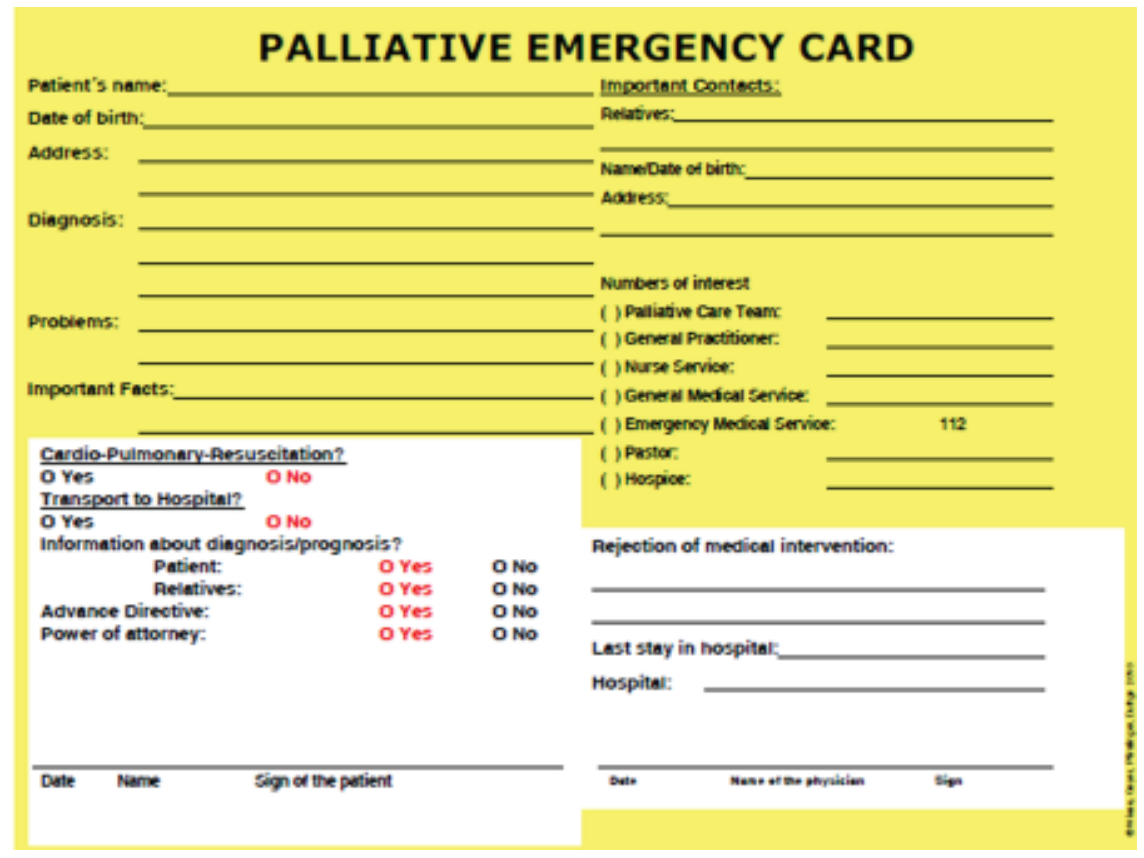

Figure I. Palliative emergency sheet (adapted to the German law). 


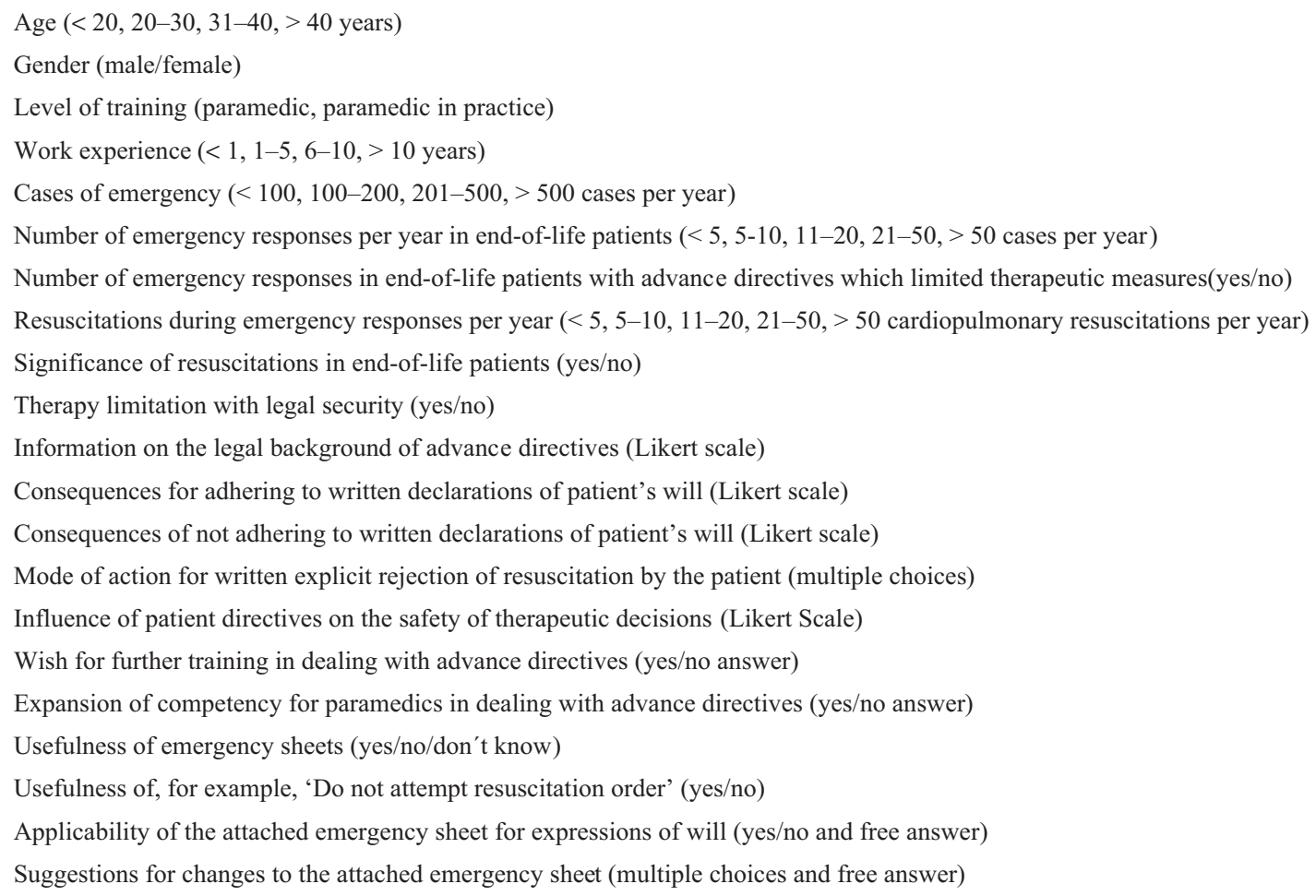

Figure 2. Items used in the questionnaire.

terminating resuscitation, as well as to examine reports of their practical experiences with advance directives and special PCCs. The impact and practicability of such advance directives in prehospital emergency medical treatment of patients at the end of life by paramedics will also be discussed.

\section{Methods}

The study design was a prospective questionnaire-based trial. Within a period of six months (April to September 2008), 900 paramedics were included in the investigation. The study was conducted in two cities: Hamburg and Goettingen, Germany. Both cities are primary based on an Emergency Medical System (EMS) which is provided by a Fire Department. Using these two cities we were able to compare the EMS in a city with a resident population of more than $1,500,000$ and a small city with a resident population of about 120,000 . A written survey was given to paramedics of all prehospital emergency fire departments of the two cities. The questionnaire was designed for selfresponse ('self-administered survey'). The survey instrument was piloted among five paramedics to determine readability and appropriateness of content. Modifications did not have to be made after the test phase (Figure 2).

For the answers/tasks/preparation of the questionnaire, total numbers, yes/no/don't know, multiple choices, free answers, and five-point Likert scales were used (so-called 'mixed methods design'). Survey respondents rated preparation as $1=$ very well, $2=$ well, $3=$ somewhat prepared, 4
$=$ poorly, and $5=$ very poorly. The tasks were rated as $1=$ very secure, $2=$ secure, $3=$ insecure, and $4=$ very insecure.

The first part of the questionnaire gathered demographic information about each responder's gender, age, years of experience as a paramedic, and current work assignments (number of emergency medical missions per year).

Participants were questioned as to (1) how often they treated palliative care patients during out-of-hospital emergency situations (so-called 'palliative emergencies'; modified according to Quest et al. ${ }^{9}$ ); (2) their experiences concerning therapeutic limitations due to the existence of an advance directive; (3) number of resuscitations per year; (4) personal assessment of the appropriateness of resuscitation (mechanical/pharmacological); (5) participants' own experiences concerning therapy limitating decisions during resuscitation; (6) participants' legal knowledge about advance directives and palliative emergency sheets; (7) possible legal consequences when paramedics follow or do not follow the patients' wishes, which are described by an advance directive; (8) certainty about and compliance with advance directives and participants' therapeutic decisions; (9) training in end-of-life care, including how to verify advance directives; (10) their wishes for expanded competency in the handling of advance directives and patient wills; (11) the applicability, meaningfulness and suitability of emergency PCCs for emergency care in palliative care patients; and (12) their suggestions for improvement or changes in the emergency sheet included (see Figure 1) for optimization of the emergency situation. 
An example of a PCC was included in the questionnaire. For each palliative care task, the respondent's answers were compared with the following characteristics (so-called independent variables: gender, age, practical experience as paramedic ( $\leq 10$ years vs. $>10$ years), number of emergency cases per month $(\leq 100$ cases vs. $>100$ cases $)$, palliative emergencies per year ( $\leq 10$ cases vs. $>10$ cases $)$, and resuscitation experiences (number of resuscitations per year).

The following palliative care tasks (dependent variables) were statistically analysed:

- Assessment of whether it makes sense to resuscitate patients in end-of-life situations subjective actions in the case of a patient's explicit rejection of resuscitation measures through an existing advance directive available to emergency staff

- Responder's knowledge of legal issues pertaining to advance directives

- Request for continuing education on the legal issues connected with advance directives and medical procedures in end-of-life patients

- The responder's sense of his or her own competency in the context of advance directives

- Assessment of the sense and applicability of the PCC (Figure 1)

As an additional parameter, participants were asked to assess a palliative emergency sheet included with the questionnaire and provide suggestions for improvements or changes. They were also asked about their own apprehensions as to possible legal consequences for therapeutic actions during the emergency situation done either in accordance with or contrary to the written advance directives. Furthermore, the participants were asked about their wishes for more training on the treatment of palliative and end-of-life patients.

\section{Participants}

In this investigation paramedics of all emergency departments of both cities were included. All of them had been actively involved in the treatment of prehospital emergencies. The participants consented to return the questionnaire within a set investigation time. Questionnaires that were sent in after this time were not included in the analysis. For the assessment of the palliative emergency sheet a copy was handed out to the participants along with the questionnaire. No commentary or presentation of the questionnaire was given by the examiner in order to avoid creating any biases in the responders.

\section{Statistical analysis}

Data from both cities were combined in a common database to ensure identical coding and analysis. The data were recorded with the MS Excel 2003 calculation program (MS Excel, Microsoft Inc ${ }^{\circledR}$, Seattle WA, USA). Descriptive statistics were used for respondent and palliative and emergency care characteristics. SPSS version 16.0 (SPSS Inc $^{\circledR}$, Chicago, Illinois, USA) was used for statistical analysis where necessary. The $t$-test and Chi-square test were used to compare descriptive proportions. Group analysis in accordance with the defined group parameters (for example the relationship between years of experience and compliance with advance directives) was assessed using the ANOVA (intergroup comparison) and the 'Posthoc-Bonferroni' test (intragroup comparison). A $p$-value of $p<0.05$ was considered to be statistically significant. The significance level for corrected $p$-value (where necessary) was designated as $p<0.05$. Descriptive values of variables were expressed as mean $\pm \mathrm{SD}$, range, median, and percentages.

The appropriate data protection guidelines and ethical principles were adhered to according to $\$ 26$ of the Declaration of Helsinki. The institutional review board approved the study (University of Goettingen and University of Regensburg) and approval was also granted by the local ethic commission (University of Goettingen and University of Regensburg). Data were collected anonymously (no personal data were collected). Anonymity was guaranteed by not numbering the questionnaires.

\section{Results}

In the defined investigation period, $728(N=728)$ paramedics responded to the questionnaire (return rate $81 \%$ : for Hamburg 620 out of $750=83 \%$; for Göttingen 108 out of 150 $=72 \%$ ). Most of the responders were male $(98.5 \%)$. Genderspecific, statistically significant differences were not found $(p>0.05)$. There were also no statistically significant differences for age and for the dependent variables $(p>0.05)$. At the time of investigation all responders were actively working in prehospital emergency medical care (EMS). The demographics of the participants are shown in Table 1.

No statistically significant differences were seen between the groups of either city investigated for the responses to the individual items $(p>0.05)$, and thus the results are given for the entire group of participants without any differentiation between the two locations. Since there were also no statistically significant differences in the biometric data for the entire group of paramedics questioned, the results can be considered representative for the entire population. Descriptive data are shown in Table 2.

\section{Consideration of the dependent variables}

Appropriateness of resuscitation in patients at the end of life (Table 3). A total of 613 participants considered resuscitation of patients at the end of life to be inappropriate ( $84 \%$ of all respondents). The significant differences between the defined groups which were worth mentioning are shown in Table 3. A statistically significant number of respondents, who were already involved in emergency cases with treatment 
Table I. Demographic data of the respondents and independent variables of the investigation (total numbers and percentages).

\begin{tabular}{|c|c|}
\hline & Total number $(\%)$ \\
\hline \multicolumn{2}{|l|}{ Age (years) } \\
\hline$<20$ & $10(1.4)$ \\
\hline $20-30$ & $185(25.4)$ \\
\hline $31-40$ & $264(36.3)$ \\
\hline$>40$ & $269(36.9)$ \\
\hline \multicolumn{2}{|l|}{ Gender } \\
\hline Male & $717(98.5)$ \\
\hline Female & $\mathrm{II}(\mathrm{I} .5)$ \\
\hline \multicolumn{2}{|c|}{ Professional qualification } \\
\hline Paramedic & $704(86.7)$ \\
\hline Paramedic in practice & $24(3.3)$ \\
\hline \multicolumn{2}{|c|}{ Professional experience as paramedic (years) } \\
\hline$<10$ & $331(45.7)$ \\
\hline$>10$ & $397(54.3)$ \\
\hline \multicolumn{2}{|c|}{ Emergency responses (per month) } \\
\hline$\leq 100$ & $430(59.1 \%)$ \\
\hline$>100$ & $298(40.9 \%)$ \\
\hline \multicolumn{2}{|c|}{ Emergency responses in palliative care patients (per year) } \\
\hline$\leq 10^{\circ}$ & $313(42.9 \%)$ \\
\hline$>10$ & $415(57.1 \%)$ \\
\hline \multicolumn{2}{|c|}{ Emergency responses with therapy limitations due to existing advance directives } \\
\hline yes & $522(71.7 \%)$ \\
\hline no & $206(28.3 \%)$ \\
\hline \multicolumn{2}{|c|}{ Resuscitation experience (number of resuscitations per year) } \\
\hline$\leq 10$ & $388(53.4 \%)$ \\
\hline$>10$ & $340(46.6 \%)$ \\
\hline
\end{tabular}

Table 2. Descriptive data of the questionnaire (total numbers and percentages).

\begin{tabular}{lc}
\hline & Number of participants (\%) \\
\hline $\begin{array}{l}\text { Feelings about own level of knowledge or the legal validity of advance directives } \\
\text { very good }\end{array}$ & $32(4 \%)$ \\
good & $116(16 \%)$ \\
satisfactory & $183(25 \%)$ \\
poor & $324(45 \%)$ \\
very poor & $73(10 \%)$ \\
Feelings about own therapy security in emergency situations in which there are & \\
existing advance directives & $33(4 \%)$ \\
very secure & $140(19 \%)$ \\
secure & $316(43 \%)$ \\
insecure & $58(8 \%)$ \\
very insecure & \\
Wish for further training in palliative emergency cases & $492(68 \%)$ \\
yes & $236(32 \%)$ \\
no & \\
Assessment of the usefulness of a special emergency sheet as therapy reference & $562(77 \%)$ \\
useful & $166(23 \%)$ \\
not useful & \\
Respondents' wishes for changes in the palliative emergency sheet presented & $563(77 \%)$ \\
Reference to the validity for paramedics & $409(56 \%)$ \\
Routine confirmation of validity through the patient/caretaker & $85(12 \%)$ \\
Copy of identification on the back side of the emergency sheet for identification purposes &
\end{tabular}




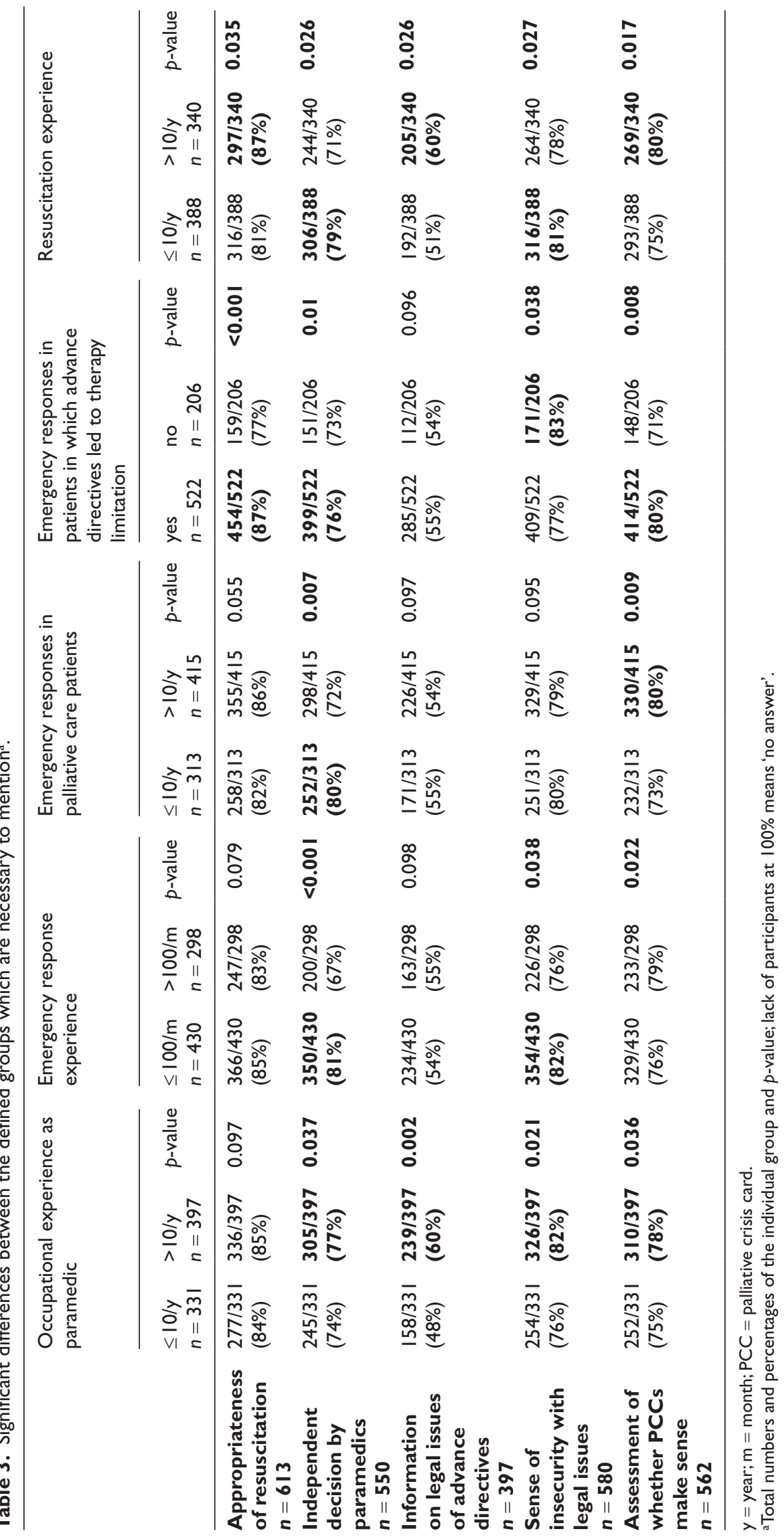


limitations and/or who were involved in more than 10 resuscitations a year, considered resuscitation in patients at the end of life as senseless.

Independent decision by paramedics to withhold resuscitation in case of legal opportunity (Table 3). Five hundred and fifty respondents (76\% of all respondents) acknowledged that resuscitation is limited or not even begun in a secure legal context for paramedics, in combination with a written advance directive or a DNAR order/PCC. A statistically significant number of responders with the following levels of professional experience stated that they would independently limit resuscitation, if given the appropriate legal security: Those with professional experience of more than 10 years; those with less than 100 emergency cases per month; responders who were involved in less than 10 emergency responses in palliative care patients per year; those who participated in emergency situations in patients with therapy limitations due to existing advance directives; as well as those who were involved in less than 10 resuscitations per year.

Information on legal issues of advance directives (Table 3). Three hundred and ninety-seven respondents (54\% of all respondents) admitted that they were poorly to very poorly informed about the legal questions in regard to advance directives. The following statistically significant number of responders considered themselves to be poorly informed about the validity of advance directives: those with experience of more than 10 years and those who were involved in an average of more than 10 resuscitations per year.

A sense of insecurity with legal issues in connection with advance directives (Table 3). Five hunded and eighty responders ( $80 \%$ of all respondents) felt only satisfactory to very insecure about legal questions in connection with advance directives. The following responders felt statistically significant less secure in dealing with advance directives in an emergency situation: those with a professional experience of more than 10 years; those with less than 100 emergency responses per month, those who were not involved in emergency cases in which a therapy limit had been set by existing advance directives, and those who were involved in less than 10 resuscitations per year.

Assessment of whether PCCs make sense (Table 3). Five hundred and sixty-two of the respondents $(77 \%$ of all respondents) considered the palliative emergency sheet (PCC) to be expedient as a short, two-page advance directive and guideline for the emergency situation. The following respondents rated the emergency sheets to be statistically significantly more useful for decision making in emergency situations: those with professional experience of more than 10 years; those with more than 100 emergency cases per month; those who were involved in more than 10 emergency responses in palliative patients per year; those who were involved in emergency responses in palliative care patients with therapy limitations due to advance directives; and those involved in an average of more than 10 resuscitations per year.

\section{Discussion}

The present study shows that paramedics feel that there are many problems for themselves in the treatment of palliative emergencies in Germany (e.g. resuscitation and legal questions). Most respondents to the questionnaire reported that they routinely deal with end-of-life patients during prehospital emergency care situations and are also routinely confronted with advance directives. However, the respondents also expressed much insecurity on legal issues in regard to the validity of advance directives in the prehospital emergency situation as well as to the obligation to comply with documented declarations of will. Related problems were already described in an international report in 1999 by Canadian paramedics. ${ }^{10}$ Such insecurity in regard to legal issues on the part of paramedics is comparable to that found in prehospital emergency physicians as well, and has been confirmed in international publications on American and Canadian paramedics. ${ }^{11-13}$

In accordance with regulations for prehospital emergency medical care in Germany, paramedics have to start mechanical and pharmaceutical resuscitation measures in the absence of a prehospital emergency physician (in accordance with the recommendations of the European Resuscitation Council ${ }^{14}$ ) in patients, even if these explicitly reject resuscitation in a written expression of will (e.g. in the form of an advance directive). At present, advance directives are being used in Germany to formulate medical measures and therapeutic limits. ${ }^{15}$ Owing to the most recent amendment to the guardianship law effective since 1 September 2009, the instruments 'advance directive' and 'power of attorney' in health care were sustainably strengthened (see paragraphs 1901 a, b and 1904 of the German code of law [BGB]). However, there are still problems in the implementation of prehospital emergency medical situations. Paramedics often find themselves in prehospital emergency situations in which an advance directive exists but it is almost impossible for them to acting in accordance with it. In such situations, there may be an ethical conflict that not only impedes the professional activity, but also can pose a challenge to personal ethical and moral concepts. Ideally, the prehospital emergency medical team (paramedics and prehospital emergency physicians) should not to be called by caregiving relatives during the expected dying situation at home. If such were the case, the problems expressed here would not occur. ${ }^{10}$ Unfortunately, the reality of this situation is different in Germany; prehospital emergency medical teams are increasingly called to care for end-of-life patients. ${ }^{16} \mathrm{~A}$ similar situation regarding advance directives could also be described for the United States. ${ }^{17}$ There is legislation in 38 states which declares the patient's declaration of will to be binding. Nevertheless, a strong 
variation in the compliance with such documents by paramedics was already reported in the 1990s. ${ }^{18,19}$

End-of-life patients who have drawn up an advance directive expect their will to be adhered to in an emergency situation, even by omitting therapeutic measures. ${ }^{20}$ In this context it is not important to the patient that it is either a paramedic or a physician who respects their will and, for example, does not begin resuscitation. The difficulties illustrated here show that advance directives do not really serve their actual purpose (as described nationally and internationally), for example, to assure the patient's autonomy at the end of his or her life. ${ }^{21}$

The paramedics surveyed here were shown a copy of a special palliative crisis card (PCC). Most of the respondents to the study rated this PCC as useful for the expression of patients' preferences at the end of life and thus as an important therapeutic aid in palliative emergency situations. However, most respondents expressed the wish that a clear statement be made that such a special advance directive for the emergency situation be legally binding.

The PCC in general is an instrument for a patient's selfdetermination, which contains important, but at the same time, short and concise written agreement on wishes for therapy, therapy limitations, contact addresses and telephone numbers of next of kin and of services and persons involved the patient's treatment. ${ }^{3}$ The PCC represents a condensed two-page advance directive reduced to the most important therapeutic arrangements (e.g. the wish for resuscitation). It offers each patient the chance to express his or her wishes for treatment (for instance, therapy limitations) beforehand. At the same time it is also important for the patient to name a person who will hold the power of attorney for health care decisions. ${ }^{3}$ The PCC thus conforms to the legal requirements for an advance directive (see paragraph 1901a of the German Civil Code [BGB]). The insecurities of the paramedics expressed by the survey participants about the legal binding of advance directives could be lessened through a concise emergency sheet and through additional training, which was wished for by most of the survey participants. According to our findings, and taking into account all parameters investigated here, training but also occupational experience had a statistically significant influence on the paramedic's deliberations as to therapy for palliative emergency patients or end-of-life patients (particularly in regard to resuscitation for such patients). With these 'experienced' respondents it was clear that they, at least theoretically, would not begin resuscitation in end-of-life patients when they considered themselves in a defined and legally secure situation. Internationally, these possible modes of action are consistent with the demands on paramedics to abstain from resuscitation measures or the continuation of such in defined situations. ${ }^{17,22}$

The paramedics surveyed in this study found special situational directives to be helpful (e.g. the shown PCC and/or so-called DNAR orders). In this context a special palliative emergency sheet like the PCC in Germany, but also other internationally established emergency protocols for end-oflife patients, provide possible model solutions. ${ }^{3,12,23}$
The present study shows that legislation in 2009 on the validity of advance directives for emergency medicine and particularly for paramedic decision-making was not adequate by far. Since September 2009 in Germany a new law concerning advance directives has come into being. This law came into force after this survey was undertaken, whereby the legal situation at the time of our investigation was different from that afterwards. However, the problems concerning the new law seem to be comparable with the old one. These dictates often contradict the wishes of end-oflife patients who have established directives so that they will not receive resuscitation and therefore allow death to take its natural course. In this regard our survey found much insecurity among respondents. In Germany demands for, or the realization of, an emergency therapy which is aided by the patient's relatives after an emergency call should also be included in future emergency and palliative care as well as ethical and legal discussions. ${ }^{12}$

\section{Limitations of the study}

The present investigation has several important limitations. First, there were no attempts to follow up respondents, and there was no comparison of respondents to non-respondents. Second, participants' responses may not accurately represent general knowledge and opinions. Third, because participation was voluntary, the participants may represent those who have strong opinions about the issues or may represent those with more time available to participate in the survey. However, the high response rate does not indicate a trend of this kind. Fourth, the questionnaire was self-administered and the validity of our instrument was not tested. Our questionnaire included some very sensitive issues on ethical actions, and the responses also could also be influenced by personal experiences (for instance from familial situations). Subjective components in the responses can therefore not be ruled out. Moreover, responding to theoretically possible modes of action are only to a limited extent possible in practical therapeutic measures, and thus our survey represents a theoretically current status but not a true evaluation of practical actions. To what extent the described measures can be transferred to a real practice situation by the respondents remains speculative and thus requires further clinical study.

\section{Conclusions}

End-of-life treatment decisions can be a special challenge for every paramedic in an emergency situation. More dialogue is necessary in the area of emergency medicine to discuss decision criteria for the care of palliative patients and to provide possible guidelines. Such guidelines were urgently wishedfor by the respondents of the present survey. Special conditions should be created for paramedics to deal with dying patients, and advance directives should be made legally binding as the new legal regulations on advance directives suggest (these regulations came into force after the present 
investigation was conducted). However, concerning the new regulations, advance directives are clearly binding in cases in which they are applicable to the respective situation. Furthermore they are only binding for physicians and not for paramedics. That's why the described problems persist at the moment. The present study was able to underscore the uncertainty in decision-making for questions of therapy limitation at end of life when advance directives are in place. The demands of previous national and international studies in regard to the importance of improved training of paramedics in end-of-life care could again be confirmed.

\section{Acknowledgements}

The authors are grateful to all of the emergency services staff who participated in the investigation. We would also like to thank Prof. Dr. G. Duttge (Center for Medical Law, University of Göttingen) for his legal advice, without which this article would not have been written, as well as his collaboration on the palliative emergency sheet presented here.

\section{Funding}

This research received no specific grant from any funding agency in the public, commercial, or not-for-profit sectors.

\section{Conflict of interest}

The corresponding author and all other authors assure that no conflicts of interest exist in connection with this work. Parts of this work were presented at the German Palliative Congress in 2010 in Dresden. This work also contains data from a doctoral thesis (MT).

\section{Authors' contributions}

MT, CHRW, CLL, and GGH participated in designing the study. MT, NM and SK participated in collecting and entering the data. BMG, AS, TK, DBR, CHRW, and GGH supported the editing of the manuscript. BMG and AS co-wrote the manuscript and added important comments to the paper. All authors read and approved the final manuscript.

\section{References}

1. Mona M. Wille oder Indiz für mutmaßlichen Willen? Ethik Med 2008; 20: 248-257.

2. Beauchamp TL and Childress JF. Principles of biomedical ethics. 2nd ed. New York: Oxford University Press, 1983.

3. Wiese CHR, Bartels UE, Geyer A, Duttge G, Graf BM and Hanekop GG. The Goettingen palliative emergency card: improvement of emergency medical care for ambulatory palliative care patients. The "yellow card for rescue services". Dtsch Med Wochenschr 2008; 133: 972-976.

4. Emanuel LL. Advance directives. Annu Rev Med 2008; 59: 187-198.

5. Crane MK and Wittink M. Respecting end-of-life treatment preferences. Am Fam Physician 2005; 72: 1263-1268.
6. Pauls MA and Ackroyd-Stolarz S. Identifying bioethics learning needs: a survey of Canadian emergency medicine residents. Acad Emerg Med 2006; 16: 645-652.

7. Roglmeier $\mathrm{J}$ and Lenz $\mathrm{N}$. The new advance directive. In: Roglmeier/Lenz 'Advance directive'; ISBN-10: 3448083585. Munich: Rudolf Haufe Verlag, 2010.

8. Simon A. Advance directives in intensive care and emergency medicine. Intensivmed 2009; 47: 43-48.

9. Quest TE, Marco CA and Derse AR. Hospice and palliative medicine: new subspecialty, new opportunities. Ann Emerg Med 2009; 54: 94-102.

10. Guru V, Verbeek R and Morrison LJ. Response of paramedics to terminally ill patients with cardiac arrest: an ethical dilemma. CMAJ 1999; 161: 1251-1254.

11. Gerth MA, Kettler D and Mohr M. Advance directives in the prehospital setting - emergency physicians' attitudes. Anaesthesiol Intensivmed Notfallmed Schmerther 2005; 40: 743-749.

12. Mengual RP, Feldman MJ and Jones GR. Implementation of a novel prehospital advance directive protocol in southeastern Ontario. Can J Emerg Med 2007; 9: 250-259.

13. Stone SC, Abbott J, McClung CD, Colwell CB, Eckstein M and Lowenstein SR. Paramedic knowledge, attitudes, and training in end-of-life care. Prehosp Disaster Med 2009; 24: 529-534.

14. European Resuscitation Council (2010) European Resuscitation Council Guidelines for Resuscitation 2010. Resuscitation 81, Suppl 1: e1-e330.

15. Bockenheimer-Lucius G. Insecureness with advance directives. Ethik Med 2003; 15: 302-306.

16. Wiese CH, Bartels UE, Marczynska K, Ruppert D, Graf BM and Hanekop GG. Quality of out-of-hospital palliative emergency care depends on the expertise of the emergency medical team-a prospective multi-centre analysis. Support Care Cancer 2009; 17: 1499-1506.

17. Marco CA. Ethical issues of resuscitation: an American perspective. Postgrad Med J 2005; 81: 608-612.

18. Ebell MH, Doukas DJ and Smith MA. The Do-Not-Resuscitation Order: a comparison of physician and patient preferences and decision. Am J Med 1991; 91: 255-260.

19. Seckler AB, Meier DE, Mulvihill M and Paris BE. Substituted judgement: how accurate are proxy predictions? Ann Int Med 1991; 115: 743-745.

20. Thorevska N, Tilluckdharry L, Tickoo S, Havasi A, Amoateng-Adjepong Y and Manthous CA (2005) Patients' understanding of advance directives and cardiopulmonary resuscitation. J Crit Care 2005; 20: 26-34.

21. Brink P, Smith TF and Kitson M. Determinants of do-notresuscitate orders in palliative home care. $J$ Palliat Med 2008; 11: 226-232.

22. Marco CA and Schears RM. Prehospital resuscitation practices: a survey of prehospital providers. J Emerg Med 2003; 24: 101-106.

23. Duttge G. Juridical problems concerning advance directives. Intensiv- und Notfallbehandlung 2005; 30: 171178.

24. German Medical Association. Recommendations concerning advance directives. Dtsch Arztebl 2010, 107: A877-882 